\title{
Malignant Uterine Ligament Neoplasm
}

National Cancer Institute

\section{Source}

National Cancer Institute. Malignant Uterine Ligament Neoplasm. NCI Thesaurus. Code C126498.

A primary or metastatic malignant neoplasm that affects the uterine ligament. 